\title{
Emergence of Hierarchy on a Network of Complementary Agents
}

\author{
M. Copelli ${ }^{1 *}$ R. M. Zorzenon dos Santos $^{1}$ and J. S. Sá Martins ${ }^{2}$ \\ ${ }^{1}$ Instituto de Física, Universidade Federal Fluminense \\ Av. Litorânea, s/n - Boa Viagem, Niterói, RJ, Brazil \\ ${ }^{2}$ Colorado Center for Chaos and Complexity/CIRES and Department of Physics \\ University of Colorado, CB 216, Boulder, CO, 80309, USA
}

October 25, 2018

\begin{abstract}
Complementarity is one of the main features underlying the interactions in biological and biochemical systems. Inspired by those systems we propose a model for the dynamical evolution of a system composed by agents that interact due to their complementary attributes rather than their similarities. Each agent is represented by a bit-string and has an activity associated to it; the coupling among complementary peers depends on their activity. The connectivity of the system changes in time respecting the constraint of complementarity. We observe the formation of a network of active agents whose stability depends on the rate at which activity diffuses in the system. The model exhibits a non-equilibrium phase transition between the ordered phase, where a stable network is generated, and a disordered phase characterized by the absence of correlation among the agents. The ordered phase exhibits multi-modal distributions of connectivity and activity, indicating a hierarchy of interaction among different populations characterized by different degrees of activity. This model may be used to study the hierarchy observed in social organizations as well as in business and other networks.
\end{abstract}

PACS numbers: 02.50.Le, 05.65.+b, 87.23.Ge

\section{Introduction}

In the last years great attention has been paid to the study of different complex systems which share the common feature of organizing themselves in networks. Examples include social networks [1, 2], the Internet [3, 旬, food webs [5], metabolic networks [6], immune networks [7, 8, 9], economic networks [10, 11] and ecological networks [12]. Despite their differences, these systems may be cast in a common framework regarding the structure of their connectivity, being mostly classified as either regular, scale-free [13], small-world [14] or random networks. Scale-free networks are characterized by power law distributions, indicating there is no typical

${ }^{*}$ Corresponding author: mcopelli@if.uff.br 
number of connections per site, whereas the small-world phenomenon is associated with high clustering coefficient and distances between sites that increase only logarithmically with system size [14], often presenting exponential connectivity distributions [13.

So far, the models proposed to study the formation and dynamics of networks are mainly based on two approaches: dynamical processes taking place in static network structures and the study of the dynamics of the network structures themselves. Most of the characterization of the network structures is focused on the dynamics of aggregation of new nodes and links to a growing network. A key point in the study of such networks is that their dynamics are based on nodes which lack identity. Or, to put it correctly, their identity and therefore their mutual affinity is uniquely determined by their connectivity. For instance, scale-free networks are obtained by linking newly introduced nodes to old ones with a probability proportional to the number of links of the latter [13, 15]. In other words, it is based on a continuous growth of nodes with preferential attachment. When these complex networks are used as a substrate to study a given dynamical process, the network itself usually remains unchanged while the states associated to the nodes evolve according to pre-determined rules [14, 16, 17, 18]. A mixed case has also been considered in the framework of game theory [11], where not only the state of the nodes evolve in time, but also their connectivity. In the last case the topological changes are biased by some state-dependent rules.

Although it has been pointed out that many of the network structures mentioned above are scale-free, the assumed rich-gets-richer dynamics that leads to this kind of structure may be appropriate only for some cases [4]. It is not clear, for instance, whether biological systems typically belong to this class of networks, since the addition of new nodes does not necessarily occur continuously, and certainly not without a strong dependence on the nodes identity. Many biological systems organize themselves through pattern recognition based on molecular interactions. Molecules, natural or synthetic, are able to replicate or interact when their shapes and chemical features are complementary. Depending on the way atoms or group of atoms are distributed spatially in a molecule, it may fit into chemical "nooks and crannies" of another [19]. Thus complementarity is essential for pattern recognition interactions underlying biological and biochemical processes. It is the basis of the DNA replication mechanisms and the generation of the immune responses, among many other important biological processes. In the case of the immune system, the control of foreign and harmful elements (antigens) to the organism is based on the recognition between molecular receptors and antigenic determinants through lock-and-key interactions. Another example comes up when we think of symbiotic species in ecological networks as complementary nodes, which generate a mutual reinforcement when they interact. In this sense the role of complementarity can be extended to social and economical systems, where agents need to cooperate with other agents in order to prevail in a competing environment.

Inspired on the complementary interactions observed in the immune system and other biological and biochemical systems [19], the model we present here may be considered as a third approach to describe the formation of networks. The effective connectivity of the system in this case emerges from the dynamical interaction among the nodes, which is in turn inherently tied to their identity. Using the parlance of socio-economical models, each node is hereafter referred to as an agent. In order for each agent to guarantee its participation in the network, it needs not only to make use of its own capabilities, but it also depends on the support of its complementary peers. The maximum number of peers each agent may have is given by a neighborhood which includes all possible complementary agents within some window of tolerance. Thus, the maximum connectivity of each agent is fixed, although large in most cases. In principle all agents could be active but in effect only a fraction of them remains 
activated due to intrinsic regulation mechanisms. The intensity of the interactions depends on the current state (hereafter referred to as "activity") of the elements of the interacting pair, which will turn out to dynamically mold their connectivity distribution. When a pair matches due to the complementarity of their attributes, their activity will increase, thus increasing the possibility of new interactions. Note that this amounts to a mutually reinforcing mechanism which operates on the activities of the nodes, not on their connectivity. The appearance of new agents is introduced by diffusion of activity in agent space, strengthening agents belonging to the similarity neighborhood of the interacting agents. These new agents are chosen by generating random mutations in one of the two interacting agents. The newly added agents, however, will not survive unless they manage to maintain themselves in the network, so that chance in this case is subjected to regulation. As we shall see, the rate at which the activity diffuses in the agent space is essential for the maintenance and stability of the network. Self-regulating mechanisms also take place, preventing agents from increasing their activity too much.

Different from the approach used recently by Castellano et al. [2], where the similarities between agents play a key role in a social influence model, here we assume that complementarity is the main mechanism in establishing the interactions among agents. The question we address here is which kind of organization is generated by individuals interacting locally by complementarity, having a limited (although large) number of interacting neighbors and whose connections are dynamically established depending on their activity?

In the studies of social behavior it is very difficult to test hypotheses concerning the relationship of individual behaviors and macroscopic regularities observed. So far the main concern in social sciences, especially in game theory and general equilibrium theory [1], has been focused on the stationary states rather than the dynamical evolution of the system. Our aim in this work is to study the essential micro or local mechanisms that lead to the dynamical emergence of global regularities and properties in a social community where individuals interact due to the complementarity of their attributes.

Surprisingly, the fact that a given environment can support only finite population, referred on the literature as the ecological principle of carrying capacity, emerges naturally in our model. We also obtain highly skewed distributions of activity. If we interpret this activity as power, influence or wealth, the inequalities observed in these distributions are characteristic of the actual human society [20]. Starting with agents having the same kind of preferences and possibilities, but a unique neighborhood, the system will end up with a multi-modal distribution of activities, since the differences produced in their states after each interaction may be amplified with time, generating the so called horizontal inequality phenomenon [1].

\section{The model}

The identity of each agent is defined by a bit string with length $b$, each bit representing for instance the presence or absence of relevant attributes. To each site $i\left(i=1, \ldots, 2^{b}\right)$ of this $b$-dimensional hyper-cubic unit cell we associate a discrete variable $N_{i} \in\left\{0,1, \ldots, N_{\text {max }}\right\}$ which represents the activity of agent $i$. If $N_{i}=0$, we say that the $i$-th agent does not take part in the network, or is inactive.

At each time step a parallel update of the individual activities of the entire system is performed. The individual update of a given activity $N_{i}$ follows two steps. In the first one, the activity of agent $i$ is decreased with probability $p_{i} \equiv \min \left(1, N_{i} / N_{\max }\right)$. This factor accounts for suppression mechanisms generated by e.g. competition for leadership or resources, 
restricting the growth of an agent's activity, which is limited to a maximal value of $N_{\max }$ :

$$
P\left(N_{i}^{\prime} \mid N_{i}\right)=\left(\begin{array}{c}
N_{i} \\
N_{i}^{\prime}
\end{array}\right) p_{i}^{N_{i}-N_{i}^{\prime}}\left(1-p_{i}\right)^{N_{i}^{\prime}}, \quad N_{i}^{\prime} \leq N_{i}
$$

where $N_{i}$ and $N_{i}^{\prime}$ are the current and updated states of agent $i$, respectively, and $P\left(N_{i}^{\prime} \mid N_{i}\right)$ is the probability with which the activity of a site $i$ changes from $N_{i}$ to $N_{i}^{\prime}$ in a time step. If the agents were allowed to be indefinitely activated, they would simply accumulate activity and the system might never attain a stationary state. In this sense the limitation on each agents activity effectively amounts to the possibility of new agents being incorporated into the network, according to the rules below. In the absence of interactions the agents activity will thus monotonically decrease, on average, like $\left\langle N_{i}^{\prime}\right\rangle=N_{i}\left(1-N_{i} / N_{\max }\right)$. If $N_{i}$ eventually surpasses $N_{\max }$, the activity of the $i$-th agent is reset to zero.

The second step on the individual activity update accounts for the mutually reinforcing interaction among agents, through which activity increases 21. We say that $j$ and $i$ are perfect complementary matches if the Hamming distance $d(i, j)$ between them (the number of different bits) is $b$ (in this case we write $j=\bar{i}$ ). However, an agent $i$ can interact not only with its perfect complementary match $\bar{i}$, but also with agents that are partially complementary. We define a parameter which measures this mismatch window, so that an agent may interact with agents that have at most a number Maxfit of equal attributes, or similarities.

If agents $i$ and $j$ are coupled, they will increase the total activity by, at the most, $n_{i j}$ units, with a probability $p_{i j}$ per unit [22], where

$$
\begin{aligned}
n_{i j} & =N_{i} N_{j} \\
p_{i j} & =\frac{N_{i} N_{j}}{\left(\begin{array}{c}
N_{\text {total }} \\
2
\end{array}\right)}
\end{aligned}
$$

and $N_{\text {total }}(t)=\sum_{i=0}^{2^{b}} N_{i}(t)$. The agent to which a unit of activity is added will be similar to either $i$ or $j$ : after having chosen one of them randomly, each of its bits may be flipped, with probability $M$ per bit (eq. (4). This provision allows for a diffusion of the activity generated in agent space, which will turn out to be essential for an agent's maintenance in the network for two main reasons: 1) by diffusing their activity to a similarity neighborhood, a given pair of agents strengthen other (similar) agents with which they can further interact, thus creating a supportive environment; 2) diffusion also helps protect an agent from the suppression factor, preventing its activity from rising too fast and reaching $N_{\max } . \quad M$ will thus be hereafter referred to as the diffusion rate.

Denoting by $l_{k}$ the agent to which the $k$-th unit in the $(i, j)$ interaction will be potentially added, the dynamics can be described by the iteration of equations 15 below, for $k=$ $1, \ldots, n_{i j}$ and $\forall i<j$ such that $d(i, j) \geq$ Maxfit:

$$
\begin{aligned}
P\left(l_{k}\right) & =\frac{1}{2} M^{d\left(l_{k}, i\right)}(1-M)^{b-d\left(l_{k}, i\right)}+\frac{1}{2} M^{d\left(l_{k}, j\right)}(1-M)^{b-d\left(l_{k}, j\right)} \\
P\left(N_{l_{k}}^{\prime \prime} \mid N_{l_{k}}^{\prime}\right) & =\delta\left(N_{l_{k}}^{\prime \prime}, N_{l_{k}}^{\prime}+1\right) p_{i j}+\delta\left(N_{l_{k}}^{\prime \prime}, N_{l_{k}}^{\prime}\right)\left[1-p_{i j}\right]
\end{aligned}
$$

where $\delta$ is the Kronecker delta. Note that the activity $i$ and $j$ created remains within the pair with probability $(1-M)^{b}+M^{b}$. 
Several quantities of interest are kept track of. As one would expect, the stability of the activity of a given agent ultimately depends on the activity of its complementary agents, including perfect and slightly defective mismatches. To capture the instantaneous average correlation between agents and their complements, we therefore introduce a variable which measures the symmetry of the activation in agent space:

$$
s(t)=\frac{2^{b} \sum_{i} N_{i}(t) N_{\bar{i}}(t)-\left(\sum_{i} N_{i}(t)\right)^{2}}{2^{b} \sum_{i} N_{i}^{2}(t)-\left(\sum_{i} N_{i}(t)\right)^{2}} .
$$

Note that $s=1$ when all $N_{i}=N_{\bar{i}}$. We also compute the fraction of agents with nonzero activity $f(t)=2^{-b} \sum_{\left\{i \mid N_{i} \neq 0\right\}}^{2^{b}} 1$ and the total activity $N_{\text {total }}(t)$ (see above) of the network. The latter is very useful from the dynamical point of view to help detect when the system reaches the stationary state.

It is instructive to observe how the populations of agents with low $\left(0<N_{i} / N_{\max }<10^{-2}\right)$, intermediate $\left(10^{-2} \leq N_{i} / N_{\max }<10^{-1}\right)$ and large activity $\left(10^{-1} \leq N_{i} / N_{\max } \leq 1\right)$ change as the dynamics evolve, which gives a first coarse-grained description of how activity is allocated in the network. The fractions of active agents $\left(N_{i} \neq 0\right)$ falling into the three logarithmic bins above will be respectively called $C_{0}(t), C_{1}(t)$ and $C_{2}(t)$. Once the stationary state is reached, we study in more detail the activity and connectivity distributions of the system. We define an agent's local connectivity $k_{i}$ as the number of its interacting peers which are active (i.e. we count the number of active agents within the Maxfit-delimited region). Note that the maximal connectivity of an agent

$$
k_{\max }=\sum_{n=0}^{\text {Maxfit }}\left(\begin{array}{l}
b \\
n
\end{array}\right)
$$

is finite, but increases rapidly as the system size grows (e.g. $b=16$ yields $k_{\max }=697$ for Maxfit $=3$ and $k_{\max }=2517$ for Maxfit=4). Therefore we have an interesting situation where the number of potential connections is very large, yet much smaller than the total number of agents (thus we are far away from mean field interactions). Out of these $k_{\text {max }}$ potential individuals, an agent will dynamically choose some, depending on how much activity the group manages to gather.

In order to further characterize our analysis on the inequality of the activity distribution in a given time step, we also calculate the Gini coefficient, which is a well known index used in socio-economical studies [1, 23]. The Gini coefficient measures the inequality of the distributions by weighing the total activity (or income, or power, etc) of the activated population by the activity of the poorest elements. Compared to other measures of inequality, it has the advantage of being applicable independently of the functional form of the activity distribution (as opposed to the Pareto index, for instance [1]). Agents are sorted by their activity and the fraction $x$ of the least excited agents (i.e. the cumulative population) is computed. Denoting by $y$ the cumulative activity associated to those agents, the function $y(x)$ is called the Lorenz curve. For perfectly "egalitarian" distributions $\left(N_{i}=N, \forall i\right)$, $y_{e g}(x)=x$. The Gini index is twice the area between $y(x)$ and $y_{e q}(x)$, attaining zero for egalitarian distributions and one if a single agent has all the activity in the system. 


\section{Results}

For the sake of clarity in all the cases discussed below we have kept Maxfit $=2$ and $N_{\max }=$ $10^{3}$. In figures 1 and 2 we show the typical behavior observed for single runs for $b=8$ and $b=12$, respectively. We randomly select an initial fraction $f(0)$ of the total number of possible agents and set their activities to $1 \%$ of the maximal activity.

The dynamics exhibited for $b=8$, although interesting in its own and revealing the appearance of emergent properties, is slightly different from the dynamics observed for larger system sizes (see the results for $b=12$ below). Figure 1 1 (a) shows the behavior of the correlation $s(t)$ among complementary agents for different values of the diffusion rate $M$. For $\mathrm{M}=0$ there is a very low correlation among complementary agents, since $s$ is essentially established by the initial configuration. The activation will basically last among these initially activated agents, due to the lack of diffusion of activity in agent space. Increasing the diffusion rate $M$ from $10^{-4}$ to $10^{-1}$, we observe that the correlation initially increases, reaching a maximum value around one for $M=10^{-2}$, in which case we interpret that a network is mounted. For $M=10^{-1}$ the correlation oscillates non-periodically, indicating that a large rate of diffusion does not guarantee the stability of the network, which mounts for a while but does not last long. This last kind of behavior is reminiscent of the "global dynamical cascades" observed in a model of heterogeneous agents for economics [11].

Figures 1 (b)-(d) show the result for the fraction of active agents with low, intermediate and large activity for $M=0,10^{-4}$ and $10^{-1}$ respectively. In the first case [Fig. 11(b)], without diffusion of activity in agent space, the system does indeed reach a stationary state, with the overwhelming majority of the active agents with large activity. The fact that no intermediate- or low-activity agents are present turns out to be an indication of failure $(s \sim 0)$ of the network to self-organize hierarchically (note that agents with $N_{i}=0$ do not enter the statistics of the $\left.\left\{C_{i}\right\}\right)$. A mild amount of diffusion $\left(M=10^{-4}\right)$ is enough to help mounting a hierarchical structure, as shown in Fig.1(c). The behavior is now completely different from the previous case and we do observe the formation of a network. This is not only characterized by the large value of $s$, but also by the stratified concentrations of active agents, which stabilize in the long run. The stationary values suggest a rough balance between low, intermediate and large activity in order to sustain the structure of the network. This pattern persists up to values $M=\mathcal{O}\left(10^{-2}\right.$ ). The results for $M=10^{-2}$ (not shown) indicate the formation of a stronger and highly activated network, with the majority $(\sim 80 \%)$ of the agents with intermediate activity, and $\sim 20 \%$ with large activity, showing some hierarchy, where stronger agents are kept by a large number of intermediate-activity agents and essentially none with low activity.

For larger values of the diffusion rate, the formation of the network is destabilized, as shown for $M=10^{-1}$ in Figure 1(d). We observe oscillations in the values of $C_{0}(t)$ and $C_{1}(t)$, suggesting that the network sustains itself for a brief period. However, this balance is quickly destroyed by the rate at which activity disperses in the network, which prevents the stronger agents and their complementary partners to satisfactorily keep their dominance. The peak in $C_{1}[t \sim 1100$ in Fig. $1(\mathrm{~d})]$ indicates that the activity which the interacting agents (and their neighbors) create spreads too much and too fast in agent space, eventually destroying their prevalence and returning the network to a non-hierarchical situation, where no particular pair or group of agents dominates the game. In the following it may happen that a new dominant group emerges out of the inherent fluctuations of the model, only

to be destabilized later, in a quasi-periodic fashion. This behavior is very robust and has been exhaustively checked over many long runs for different initial configurations. For higher 

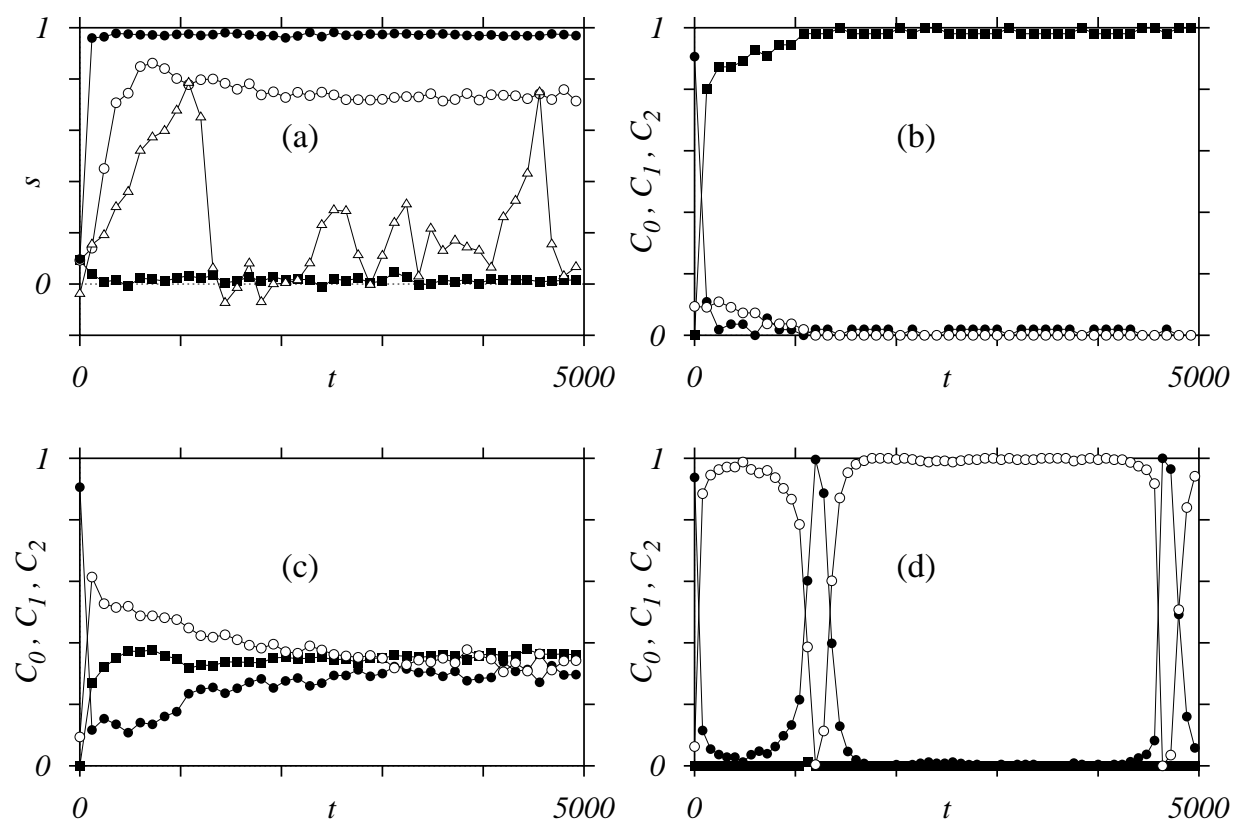

Figure 1: Typical dynamical behavior for $b=8$ with $f(0)=0.25$ ( $t$ in Monte Carlo steps): (a) the correlation $s$ among agents for $M=0$ (squares), $10^{-4}$ (open circles), $10^{-2}$ (black circles) and $10^{-1}$ (triangles). The fraction of active agents with low (open circles), intermediate (black circles) and large (squares) activity are shown from (b) to (d) for $M=0,10^{-4}$ and $10^{-1}$, respectively.

diffusion rates $(M \simeq 0.4)$, the peaks described in the previous case disappear and $s$ fluctuates around zero, indicating the complete absence of correlation among the agents. In this case $C_{0}$ is essentially one, showing that the system consists of a very large number of weakly activated (and disconnected) agents. It is interesting to point out that in the whole range $10^{-4}<M<10^{-1}$ (where the network is mounted), the fraction of active agents $f$ remains above $\sim 80 \%$ in the stationary state, indicating that the majority of agents is active.

Results for $b=12$ are shown in Figure 2 for two representative values of the diffusion rate. The first interesting feature we observe when $M=10^{-3}$ [Fig. 2(a)] is that, unlike the 8-bit case, there is a long transient period before the fraction of active agents $f$ and the correlation $s$ stabilize. After that, the emergence and establishment of the network then occur in a relatively short time. Moreover, $f$ remains around 0.2 in the plateau, signaling that the majority of the agents have zero activity and do not take part in the network. In the evolution of concentrations $\left\{C_{i}\right\}$ shown in Fig 2(c), most of the active agents have low activity, followed by agents with intermediate and large activity. The same behavior is observed for different samples, although the stationary concentrations may fluctuate around slightly different mean values. The larger 12-bit system is therefore much less excited and sparser than the smaller 8-bit one: not only is the fraction of active agents smaller, but also the activity of those agents is typically less than for the smaller system. Fig. 2(b) shows the results for a higher diffusion rate $\left(M=10^{-1}\right)$ where again we observe a long transient, after which a peak in $s$ and in $f$ occurs, corresponding to an attempt to mount the network. The attempt fails due to the instability generated by the high rate at which activity diffuses. The system will keep spiking indefinitely, similarly to what has been observed for 8 bits in the 

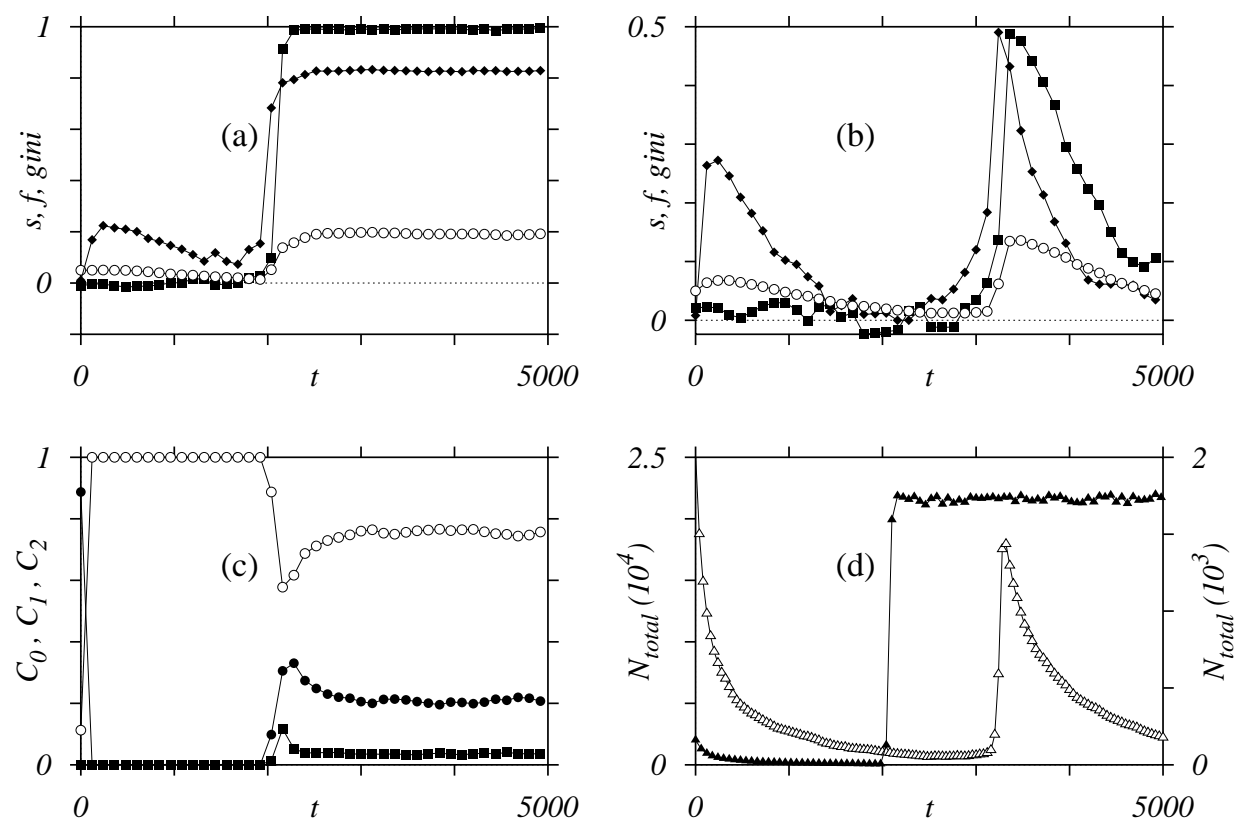

Figure 2: Typical dynamical behavior for $b=12$, starting with $f(0)=0.05$ ( $t$ in Monte Carlo steps): correlation $s$ among agents (squares), fraction of active agents $f$ (open circles) and Gini coefficient (diamonds) for $M=10^{-3}$ (a) and $M=10^{-1}$ (b); fraction of active agents with low (open circles), intermediate (black circles) and large activity (squares) for $M=10^{-3}$ (c); and the total activity of the system (d) for $M=10^{-3}$ (black triangles - left axis) and $M=10^{-1}$ (open triangles — right axis).

same parameter region. Finally, a complementary view of these phenomena can be composed by the results in Fig. 2(d), which shows the evolution of the total activity for both diffusion rates. For $M=10^{-3}$ the stabilization of $s$ and $f$ is associated with the stabilization of $N_{\text {total }}$ at a large value, while for $M=10^{-1}$, the peaks in $s, f$ and $N_{\text {total }}$ occur simultaneously, the latter attaining a much lower level (note the different vertical scales) than in the $M=10^{-3}$ case.

Finally, it is interesting to observe the behavior of the Gini coefficient for both diffusion rates. Figures. 2(a)-(b) show that it departs from zero (since the initial agents have the same activity) and immediately grows, indicating the immediate creation of inequalities due to the fluctuations. Note however that during this growth $s$ remains near zero, showing that the initial fluctuations do not immediately lead to a structuring in agent space. The inequality decreases a little and then increases, now lifting with it the correlation. The Gini coefficient then stabilizes around 0.80 , indicating a highly uneven distribution.

The diffusion rate thus plays an extremely important role in connecting the agents, its effect being: too low or too high diffusion rates deteriorate the stability of the network, while intermediate values increase the diversity of active agents in the right amount to allow new pairs to match, thus providing the mechanism for the system to adapt, incorporating new agents. Note that in the current model this is the only source providing new active agents. The parameter Maxfit plays a complementary role to that of the diffusion rate on the regulation of the network. A large diffusion rate may eventually be compensated by a 


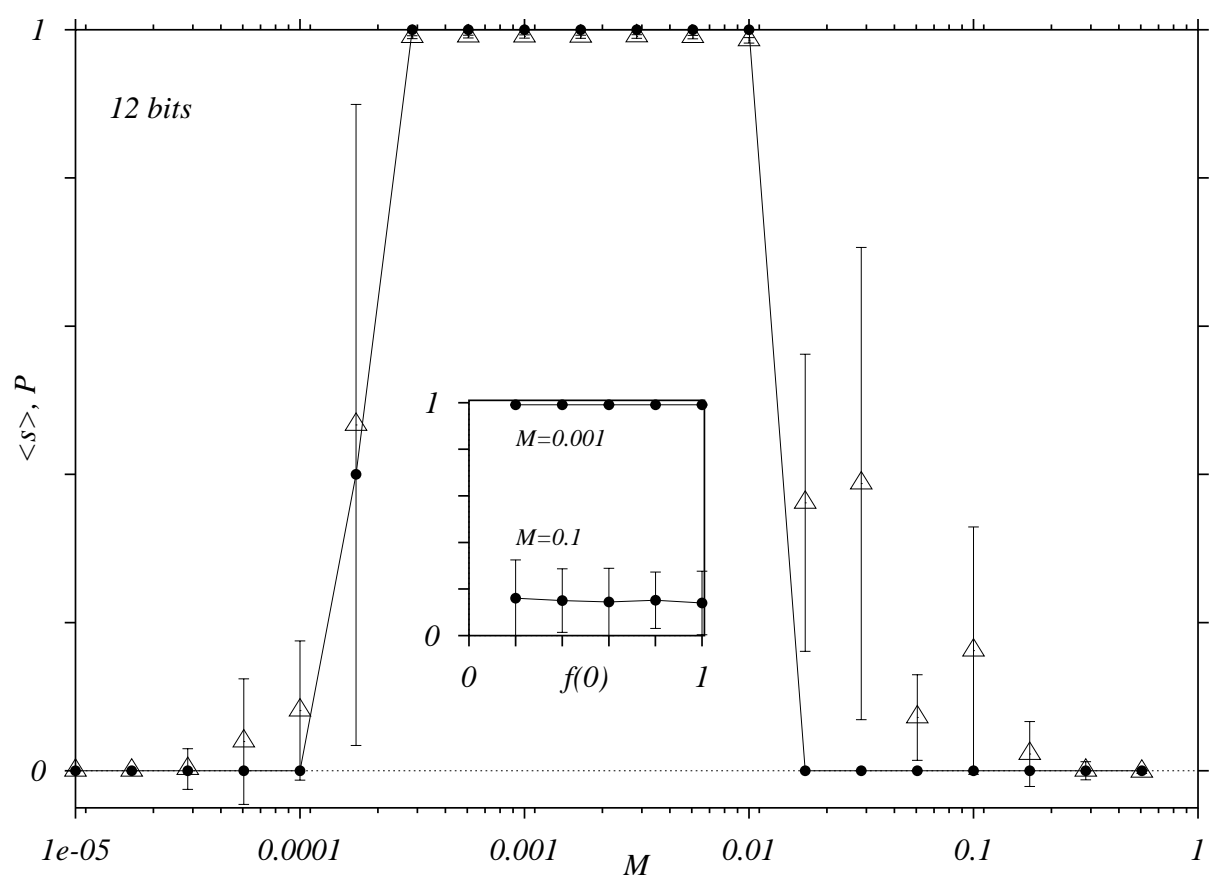

Figure 3: Time-averaged correlation $\langle s\rangle$ (triangles) and "mount probability" $P$ (black circles - see text for details) as a function of $M$ for 12 bits. Inset: $\langle s\rangle$ as a function of the initial concentration for $M=10^{-3}$ (upper curve) and $10^{-1}$ (lower curve). The results correspond to an average over 50 runs.

smaller Maxfit, which renders the agents more specific in the interaction with their mirrors (in the sense that it reduces the number of interacting peers). However, Maxfit cannot be too low lest there be not enough agents to create a supportive neighborhood, specially for low diffusion rates.

The dependence of the formation of the network for $b=12$ on the diffusion rate is summarized in Fig. 3, which shows the stationary value of $s$, averaged over 500 time steps and 50 runs, as a function of $M$. In order to obtain this plot we have adopted the following protocol: we consider that the network is mounted if $s$ stays larger than a threshold value $s_{\min }=0.8$ (estimated from many individual runs for the parameters under consideration) during a period of 1000 time steps. This time interval is long enough for an eventual peak not to be erroneously detected as a successful attempt. The "mount probability" is defined as the fraction of times that this criterion is satisfied. We observe then two transitions, one at $M \simeq 10^{-4}$ and another at $M \simeq 0.03$. The large error bars in $s$ around the transition points are the signature of the peaks observed in Fig. 2. The first transition is mainly provoked by the suppression factor: since the rate at which activity disperses is very low, the initially correlated agents reinforce themselves, leading to a fast increase in their activity which strongly submits them to the suppression factor. In this case no correlation is maintained long enough to mount the network. The second non-equilibrium phase transition is due to the dynamics of the system as discussed below.

Another interesting feature that emerges from this model is the weak dependence on the initial conditions. For several values of $M$ we have tested the evolution of the system for different initial fractions of nonzero agents. What we have observed, for both 8 and 12 
bits, is that the results are qualitatively the same for whatever initial conditions we choose, except of course for the limiting case $f(0) \simeq 0$, in which the activities eventually decay to zero due to a lack of matching pairs. This result is clearly observed in the inset of Fig. 3, which shows the stationary value of $s$ as a function of $f(0)$ for 12 bits. The mechanisms behind this independence can be understood on the basis of the transients of both $N_{\text {total }}$ and $s$ (Fig. 2). In order for the network to stabilize, the unpaired initial agents will first be "cleaned up", leaving mostly pairs that match within the margins set by Maxfit. The dynamics automatically takes care of this initial dismantling, since all those agents which do not have their matching pairs will gradually decrease their activity [see Fig. 2(d)]. This in turn increases the probability that the remaining matching agents interact, and stationarity is then achieved when activity increases and losses are balanced. This explains why the model is not very sensitive to the initial conditions. Larger initial values of $f$ only increase the time spent in the transient, therefore we have typically used $f(0)=0.05$ for 12 bits. In the 8-bit case, the initial transient is much shorter and of a different nature because the network somehow manages to accommodate a much larger fraction of agents in highly excited states, showing a different dynamics. Due to the small system size all potential pairs will be effectively connected, which is not the case for larger system sizes. This particular behavior might be related to what is observed in population models and commonly referred to as finite population effect 24].

It is also important to understand how the behavior of the system changes when the size of the space gets larger. Representative simulations for $b=16$ (using $f(0)=0.015$ ) show that the fraction of active agents $f$ in the stationary state is even smaller than for 12 bits, but the behavior of the concentrations $\left\{C_{i}\right\}$ remains qualitatively the same, indicating that those are the relevant quantities for larger system sizes. The dynamical behavior for 16 bits exhibits the same features as for 12 bits, the corresponding plots (not shown) being very similar to Fig. 2. Even though a more detailed analysis of the parameter space is currently under investigation, it is clear that the effects of the diffusion rate $M$ follow the same trends both for larger systems and for smaller systems: if it is either too large or too small, no balance can be achieved and the agents will fail to establish a connected network. It is interesting to note that for Maxfit $=2$ the absolute number of active agents in the stationary state remains $\mathcal{O}\left(10^{3}\right)$ when one goes from 12 to 16 bits, explaining the decrease in $f$. This number grows when Maxfit=3, but still corresponds to a small fraction of the $2^{b}$ potential agents, for large systems, and it is the diffusion rate that guarantees its stability. Without any direct suppression mechanism acting on the number of agents belonging to the network, the local rules of the model therefore lead to an effective "carrying capacity" of the system [1]. The proper scaling of Maxfit with the growth of the system is yet to be fully understood, i.e., how does the stationary value of $f$ depend on the relation between Maxfit and $b$ ? We also conjecture whether a larger agent space would allow the system to adapt more easily to perturbations, which normally would destabilize the network for small systems. These and other questions concerning the robustness of the network on the parameter space are currently under investigation and will be published elsewhere.

We finally turn to the characterization of the ordered phase, focusing at intermediate values of $M$. Our analysis are based on the emergent distribution of activity and connectivity in the network, which turns out to be highly non-trivial. $P(N)$ measures how activity is allocated, whereas $P(k)$ is the connectivity distribution. These distributions are calculated in the stationary state for $b=8\left(M=10^{-2}\right)$ and for $b=12$ and $b=16\left(M=10^{-3}\right)$ over 30 runs. Results are shown in Fig. 4 . For the three cases [Figs. 1(a)-(c)], we obtain a multimodal distribution of activity. Therefore, the system does not have a single typical activity, 

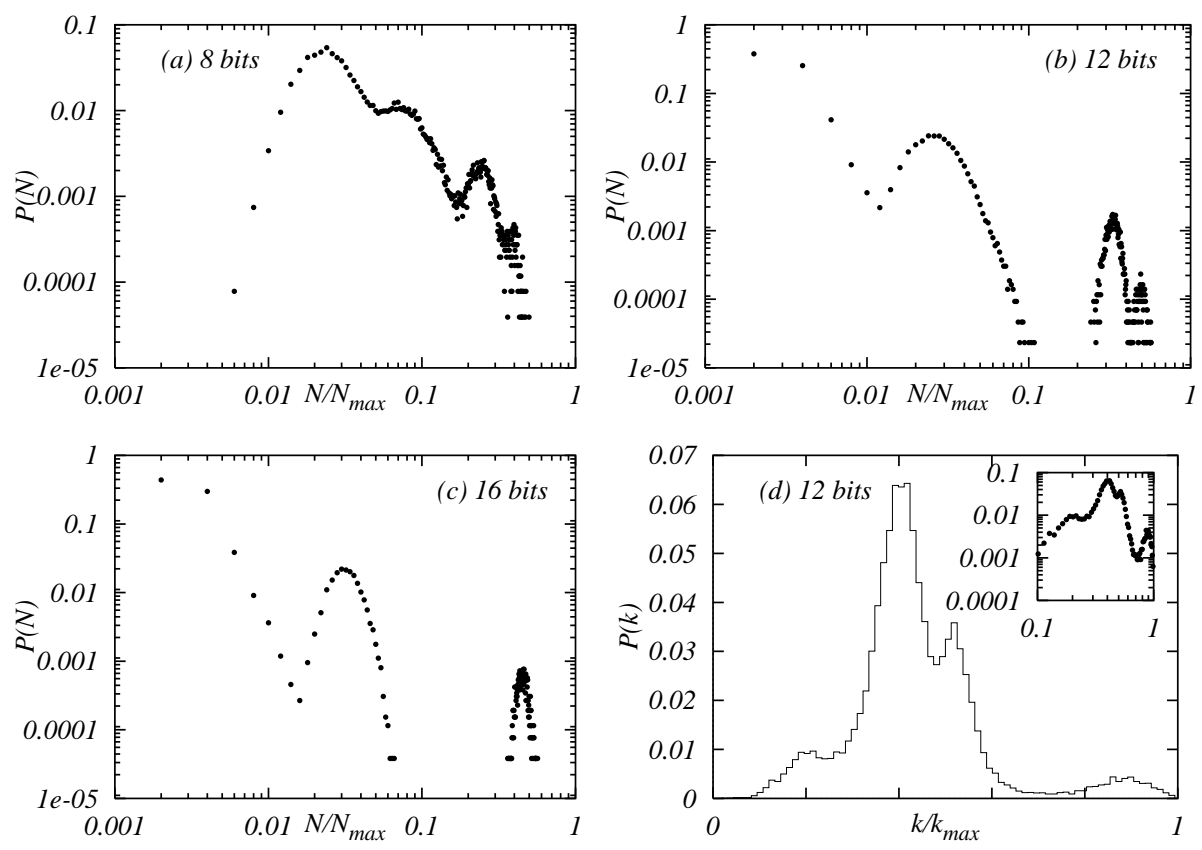

Figure 4: Activity distributions for $M=10^{-3}$ : (a) $b=8$, (b) $b=12$ and (c) $b=16$; connectivity distribution for $b=12(\mathrm{~d})$. Inset: same as (d) in log-log scale.

nor does it possess a scale-free activity distribution: as opposed to that, we observe that there are four well-defined "typical" values of activity (other than zero) for 8 and 12 bits, and three for 16 bits, defining a hierarchy in agent space. We therefore justify in hindsight the arbitrary logarithmic binning as defined by the variables $C_{0}, C_{1}$ and $C_{2}$, which are actually reasonable coarse grained descriptions of the peaks of Fig. 4. It is also worth noting that, for 12 and 16 bits, there is a gap in the activity distribution, a feature that has also been observed in larger system sizes.

The distribution presented in Fig. 4(d) exhibits an even richer behavior for 12 bits. Not only do we observe multi-modality again, but also a non-monotonic behavior of the peaks. In other words, the majority of the agents are "connected" to $\sim 40 \%$ or $\sim 50 \%$ of their potential collaborators, while two smaller groups either have $\sim 20 \%$ or $\sim 90 \%$ of their possible connections actually established. The distribution for 16 bits has similar features, while for 8 bits $P(k)$ in its most ordered regime is simply given by $P(k)=\delta\left(k, k_{\max }\right)$ (where $k_{\max }$ is defined in eq. 7), reflecting the fact that $f$ saturates at one. The increase of the variance of the activity distribution corresponds to an increase of the horizontal inequality. It depends on the rate at which new agents are activated (diffusion of activity in agent space), since new agents take some time until their activity is brought into line with those already prevailing in the system.

\section{Conclusions}

In this paper we proposed a network model of mutually reinforcing agents by using a bit-string approach. The model is inspired in the complementary interactions observed in biological and biochemical systems. The interaction among the agents is based on their identity. All 
agents have potentially the same connectivity although the actual individual connectivities are established by dynamical rules based on interactions that allow perfect and slightly defective mismatches between agents. A suppression mechanism acting upon agents limits the maximum activity that may be associated to each agent. New agents may be added to the system through the interactions among active agents, which allow the diffusion of activity in agent space. The rich dynamical behavior exhibited by this kind of network depends on the diffusion rate, the size of the mismatch window and the maximum activity allowed to each agent. The agents prospering in activity may activate new agents. For very low or very large diffusion rates there is a lack of correlation between complementary agents and the network is not formed, while for intermediate values of this parameter we observe the formation of a stable network.

For small systems ( 8 bits) we observe a highly excited network involving most of the available agents, while for larger systems this network is much sparser. However, regardless of the size of the system, we observe a hierarchical organization reflected by the multi-modal distribution of activities and connectivities. When we increase the size of the system from 12 to 16 bits, we observe that the absolute number of agents participating in the network has the same order of magnitude. Therefore the number of competent inactive agents increases as the system size grows, a feature that might favor the adaptation of large networks to perturbations.

The skewed distributions obtained correspond to the emergence of structures or selforganization in the network of activated agents, induced by local interactions between the agents. The system organizes itself in a hierarchical structure where we find grosso modo three classes: highly, intermediate an low activated agents. Since we considered the network of active agents, we do not include among the classes the non-active one. For the parameters considered in this paper the Gini coefficient stays always between $0.70-0.80$, indicating a rather unequal distribution of activities.

In the stationary state we do observe the organization of the system with a finite number of active agents, without any need of ab initio considerations about finite population. This means that at this point there is an equilibrium between agents being deactivated and those being activated. The model may explain the hierarchy observed in business networks and social networks. It may be also a good model to describe the formation of corruption networks. For corruption to take place, the interaction among the agents in this case cannot be based on similarities or acquaintances, but rather on complementarity that generates a dependence among the participants. Diffusion can then be understood as a way of sharing power in order to protect leading agents from betrayal. As another application we may consider the introduction of different types of interactions (other than mutually reinforcing), which might give rise to multi-modal distributions which could then be interpreted as the emergence of trophic levels in ecology.

Acknowledgments This work was partially supported by CNPq, CAPES and FAPERJ. We would like to thank Nestor Caticha and Américo T. Bernardes for their invaluable suggestions and criticisms.

\section{References}

[1] J. M. Epstein and R. Axtell. Growing Artificial Societies: Social Science from the Bottom Up. Brookings Institution Press, 1996. 
[2] C. Castellano, M. Marsili, and A. Vespignani. Nonequilibrium phase transition in a model for social influence. Phys. Rev. Lett., 85:3536-3539, 2000.

[3] B. A. Huberman and L. A. Adamic. Internet - growth dynamics of the world-wide web. Nature, 401:131, 1999.

[4] A.-L. Barabási, R. Albert, H. Jeong, and G. Bianconi. Power-law distribution of the World Wide Web. Science, 287:2115a, 2000.

[5] G. Caldarelli, P. G. Higgs, and A. J. McKane. Jour. Theor. Biol., 193:345, 1998.

[6] H. Jeong, B. Tombor, R. Albert, Z. N. Oltvai, and A.-L. Barabási. The large-scale organization of metabolic networks. Nature, 407:651, 2000.

[7] M. Cohn. The biology of recognitive repertoires. Immunology Today, 21(9):433-435, 2000.

[8] R. M. Zorzenon dos Santos and A. T. Bernardes. The stable-chaotic transition on cellular automata used to model the immune repertoire. Physica A, 219:1-12, 1995.

[9] R. M. Zorzenon dos Santos and A. T. Bernardes. Immunization and aging: A learning process in the immune network. Phys. Rev. Lett., 81:3034-3037, 1998.

[10] M. Paczuski, K. E. Bassler, and A. Corral. Self-organized network of competing boolean agents. Phys. Rev. Lett., 84:3185-3188, 2000.

[11] M. G. Zimmermann, V. M. Eguíluz, and M. San Miguel. Cooperation, adaptation and the emergence of leadership. In A. Kirman and J.-B. Zimmermann, editors, Economics with Heterogeneous Interacting Agents, number 503 in Lecture Notes in Economics and Mathematical Series. Springer, 2001.

[12] M. Lässig, U. Bastolla, S. C. Manrubia, and A. Valleriani. The shape of ecological networks. http://arXiv.org/abs/nlin.A0/0101026, 2001.

[13] A.-L. Barabási and R. Albert. Emergence of scaling in random networks. Science, 286:509-512, 1999.

[14] D. J. Watts and S. H. Strogatz. Collective dynamics of 'small-world' networks. Nature, 393:440-442, 1998.

[15] M. E. J. Newman. Clustering and preferential attachment in growing networks. Phys. Rev. E, 64:025102, 2001.

[16] R. Pastor-Satorras and A. Vespignani. Epidemic spreading in scale-free networks. Phys. Rev. Lett., 86:3200-3203, 2001.

[17] R. Pastor-Satorras and A. Vespignani. Epidemic dynamics and endemic states in complex networks. Phys. Rev. E, 63:066117, 2001.

[18] A. T. Bernardes, D. Stauffer, and J. Kertesz. submitted to publication, 2001.

[19] J. Rebek Jr. Synthetic self-replicating molecules. Scientific American, July:48-55, 1994.

[20] J. Persky. Retrospectives: Pareto's law. Journal of Economic Perspectives, 6:767-773, 1992.

[21] W. B. Arthur. Self-reinforcing mechanisms in economics. In P. W. Anderson, K. J. Arrow, and D. Pines, editors, The Economy as an Evolving Complex System. AddisonWesley, Redwood City, CA, 1988.

[22] Y. Togashi and K. Kaneko. Transitions induced by the discreteness of molecules in a small autocatalytic system. Phys. Rev. Lett., 86:2459, 2001. 
[23] A. Drăgulescu and V. M. Yakovenko. Evidence for the exponential distribution of income in the USA. cond-mat/0008305.

[24] A. O. Sousa, S. Moss de Oliveira, and A. T. Bernardes. Simulating inbreeding depression through the mutation accumulation theory. Physica A, 278:563-570, 2000. 